\title{
REVIEW
}

\section{Management of severe pulmonary arterial hypertension}

\author{
J-L. Vachiéry* and G. Simonneau*
}

ABSTRACT: Pulmonary arterial hypertension (PAH) is a severe, progressive condition of the small pulmonary vessels that leads to increased pulmonary vascular resistance, right ventricular failure and death. Patients in World Health Organization functional class (WHO FC) IV are the most severely affected in terms of disease severity, symptomatic impairment, exercise capacity and haemodynamics, with a very poor prognosis and low survival rate. Recent developments in PAHspecific therapies have conferred significant prognostic improvements upon PAH patients, especially when coupled with management strategies such as goal-oriented therapy and combination treatment. Despite these important developments, the outlook for WHO FC IV PAH patients remains poor. This article examines the recommendations for WHO FC IV patients that appear in current PAH treatment guidelines and the research underpinning this guidance, and discusses possible future directions for treatment of this severely unwell patient population.

KEYWORDS: Combination treatment, prognosis, pulmonary arterial hypertension, World Health Organization functional class IV

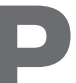
ulmonary arterial hypertension $(\mathrm{PAH})$ is a devastating, life-threatening disease with a rapid progression and poor prognosis when untreated [1-3]. Diagnosis of PAH is based on the presence of pre-capillary pulmonary hypertension $(\mathrm{PH})$, defined as an elevated mean pulmonary arterial pressure $\left(\bar{P}_{\mathrm{pa}}\right)$ of $>25 \mathrm{mmHg}$ at rest in the absence of any other potential cause, such as $\mathrm{PH}$ due to lung diseases, chronic thromboembolic PH (CTEPH) or other diseases $[4,5]$. In the classification of pulmonary $\mathrm{PH}, \mathrm{PAH}$ comprises group 1 [6], encompassing five different types of PAH with different underlying aetiologies. All display common clinical features and almost identical changes in the lung microcirculation, resulting from similar pathophysiological mechanisms involving pulmonary vasoconstriction and vascular remodelling.

Recent studies provide reliable epidemiological data that better elucidates the scope of the problem. The idiopathic form (IPAH) has an annual incidence of 2.4-6 per million of population, and prevalence rates of $15-50$ cases per million $[5,7,8]$. Idiopathic and heritable PAH (with or without an identifiable genetic mutation) represent $\sim 50 \%$ of the cases seen at PAH clinics [7]. In the remaining 50\%, $\mathrm{PAH}$ is found in association with another underlying condition or risk factor (APAH). An important clinical subgroup is $\mathrm{PAH}$ associated with connective tissue diseases (PAH-CTD), which has poorer prognosis than IPAH. Overall, the prevalence of PAH-CTD has been estimated to be $2.3-10$ cases per million in the general population [7-9]. Between 7\% and $12 \%$ of patients with systemic sclerosis (SSc) and around $0.5-14 \%$ of patients with systemic lupus erythematosus are reported to have $\mathrm{PAH}$, making these the two most frequent causes of PAHCTD [10-12]. Patients with HIV infection and portal hypertension may also develop PAH in $<1 \%$ of cases. Little is known on the epidemiology of other forms of APAH $[5,6]$.

The severity of PAH is determined according to the classification of the New York Heart Association (NYHA), initially developed for heart failure and then modified by the World Health Organization (WHO) for PAH. The basis of the NYHA classification is to link symptoms (dyspnoea and fatigue) with activity limitations. The determinant is the level of physical activity that the individual patient can carry out without symptoms. In the WHO functional classification (WHO FC), syncope and signs/symptoms of right heart failure were added to dyspnoea and fatigue in order to reflect the severity of the disease (table 1) [13, 14]. It is important to recognise

\section{AFFILIATIONS}

*Pulmonary Vascular Diseases and Heart Failure Clinic, Dept of Cardiology, CUB-Hôpital Erasme, Brussels, Belgium.

\#Dept of Pneumology and ICU, National Reference Center for Pulmonary Hypertension, South Paris University, Hopital Antoine Béclère, Paris, France.

CORRESPONDENCE

J-L Vachiéry

Pulmonary Vascular Diseases and Heart Failure Clinic

Dept of Cardiology CUB-Hôpital Erasme 808 route de Lennick 1070 Brussels Belgium

E-mail: jean-luc.vachiery@ulb.ac.be

Received:

Aug 242010

Accepted after revision:

Sept 292010

PROVENANCE

Publication of this peer-reviewed article was supported by Actelion Pharmaceuticals Ltd, Switzerland (unrestricted grant, European Respiratory Review issue 118). 
the role of presyncope or syncope as a powerful marker of prognosis in patients with PAH. Historical data show that, without treatment, patients with $\mathrm{PAH}$ have a generally poor prognosis; those in WHO FC IV have the worst prognosis of all [15]. In fact, baseline WHO FC is a strong predictor of survival, with WHO FC IV being associated with significantly poorer survival compared with WHO FC II/III in patients with IPAH and PAH-CTD [9, 16-18].

Over the past decade, increased understanding of the pathophysiology of $\mathrm{PAH}$ has led to the development of $\mathrm{PAH}$-specific therapies, which have brought about considerable prognostic improvements for patients with PAH. The management of $\mathrm{PAH}$ is continuing to evolve in an attempt to further improve outcome, with strategies including combination therapy, goal-oriented treatment algorithms and new targets for therapy of increasing interest. However, despite these improvements, evidence shows that prognosis remains very poor for the most severely affected patients in WHO FC IV. This article will discuss the current guidelines for management of WHO FC IV PAH and the evidence behind the recommendations, and discuss possible future strategies for this particularly challenging group of patients.

\section{CLINICAL PRESENTATION OF WHO FC IV}

Patients with WHO FC IV PAH are severely impaired by their disease. Exercise capacity is almost totally abolished as a result of symptoms such as dyspnoea, syncope, dizziness and signs of congestion. There is an association between symptomatic impairment, haemodynamic variables, exercise capacity and disease severity in patients with PAH. For patients in WHO FC $\mathrm{IV}$, cardiac index, both at rest and during exercise, is severely reduced $\left(<2.1 \mathrm{~L} \cdot \mathrm{min}^{-1} \cdot \mathrm{m}^{-2}\right)$, pulmonary vascular resistance

\begin{tabular}{ll} 
TABLE 1 & $\begin{array}{l}\text { World Health Organization functional } \\
\text { classification (WHO FC) definitions }\end{array}$ \\
\hline Class & Definition \\
\hline WHO FC I & Patients with PH but without resulting limitation of \\
& physical activity \\
& Ordinary physical activity does not cause undue \\
& dyspnoea or fatigue, chest pain or near syncope \\
WHO FC II & Patients with PH resulting in slight limitation of \\
& physical activity \\
& They are comfortable at rest \\
& Ordinary physical activity causes undue dyspnoea or \\
& fatigue, chest pain or near syncope \\
Patients with PH resulting in marked limitation of & physical activity \\
WHO FC III $\quad$ They are comfortable at rest \\
\\
Less than ordinary activity causes undue dyspnoea or \\
fatigue, chest pain or near syncope \\
Patients with PH with inability to carry out any physical \\
activity without symptoms \\
These patients manifest signs of right-heart failure \\
Dyspnoea and/or fatigue may even be present at rest \\
Discomfort is increased by any physical activity
\end{tabular}

PH: pulmonary hypertension.
(PVR) index is high $\left(>2,000 \mathrm{dyn} \cdot \mathrm{s}^{-1} \cdot \mathrm{cm}^{-5} \cdot \mathrm{m}^{-2}\right)$, signs of right ventricular failure are often present and 6-min walk distance $(6 \mathrm{MWD})$ is especially low $(<200 \mathrm{~m})$ [7].

Because WHO FC status is related to functional capacity, patients in FC IV are expected to have a significant impairment in quality of life (QoL) compared with those in WHO FC III. This has been supported, at least in part, by a study in which 48 patients with either PAH $(n=26)$ or CTEPH $(n=22)$ completed the Minnesota Living with Heart Failure (MLHF) QoL questionnaire [19]. A higher score, reflecting a greater perceived impairment of QoL, was achieved in FC IV patients compared with FC III; total scores 62 and 41, respectively; $\mathrm{p}<0.001$. Such a QoL questionnaire has been used in clinical trials in severe PAH and proved sensitive to changes [20]. However, the MLHF QoL questionnaire was originally designed and validated to assess QoL in heart failure. It is thus lacking specificity for PAH. More recently, the Cambridge Pulmonary Hypertension Outcome Review (CAMPHOR) scale [21] has been designed to be more specific, allowing for a better evaluation of QoL in PH. It explores three integrated dimensions: overall symptoms (comprising energy, breathlessness and mood subscales), functioning and QoL. The CAMPHOR scale has been validated in patients with $\mathrm{PH}$ according to the WHO diagnostic classification [5] and, therefore, includes patients with all forms of $\mathrm{PAH}, \mathrm{PH}$ and CTEPH. No PAH-specific QoL questionnaire has yet been developed, and the exact nature of the QoL burden associated with PAH in general, and with WHO FC IV PAH in particular, remains to be defined [21-24].

In contrast to common belief, such severely ill and difficult to treat patients represent a significant proportion of the $\mathrm{PAH}$ population, including newly diagnosed patients. Registry data show that the majority of patients with PAH are not diagnosed until their disease is in an advanced form, and up to $24 \%$ are already in WHO FC IV $[7,9,25,26]$. This is the case for all aetiologies of $\mathrm{PAH}$, including those associated with diseases known to be risk factors for the condition, such as SSc or congenital heart disease (CHD), for which screening is recommended [7].

Acute progression to right ventricular failure can occur in patients with $\mathrm{PAH}$, and often requires hospitalisation to ensure adequate monitoring and vasoactive drug provision [27]. Although guidelines for management of right ventricular failure in the absence of $\mathrm{PH}$ have been published, there are few data in patients with $\mathrm{PH}$ [28]. Recently, however, a prospective French study of 46 consecutive PAH patients admitted to a large, $\mathrm{PH}$-specific intensive care unit (ICU) for right heart failure clarified the influence of demographic, clinical and biological data and therapy on clinical outcomes [27]. Mean length of ICU stay among this mixed PAH population was 14 days, and overall mortality was high, at $41.3 \%$. Survivors and nonsurvivors did not differ at admission in terms of demographics, clinical data or last haemodynamic measurements. Although no association was found between pre-ICU WHO FC and survival status, mortality was predicted by high levels of $\mathrm{N}$-terminal pro-brain natriuretic peptide, $\mathrm{C}$ reactive protein, and serum sodium and creatinine levels at admission. During the first 3 weeks in the ICU, systemic arterial pressure was the only clinical parameter found to be 
associated with survival. Nonsurvivors exhibited a higher rate of infections during ICU hospitalisation. In terms of treatments, progressive increase in dobutamine administration rate was associated with poorer outcome [27]. Further study is required to refine clinical guidance for acute right ventricular failure in $\mathrm{PAH}$ patients.

\section{WHO FC IV IS A KEY PREDICTOR OF PROGNOSIS}

The natural prognosis in patients presenting with WHO FC IV $\mathrm{PAH}$ at diagnosis is poor. In untreated IPAH, median survival is only 6 months for patients in WHO FC IV, compared with 2.5 yrs for those in WHO FC III, and 6 yrs for those in WHO FC I and II [15]. This rapid progression is associated with the decline in right ventricular function seen when patients progress from a symptomatic but relatively stable status in WHO FC II and III, to rapidly progressive WHO FC IV. Recent, robust evidence suggests that such a dismal prognosis for FC IV remains the case, even for patients receiving targeted therapies. In fact, WHO FC is one of the strongest predictors of outcome, whether at baseline or under medical therapy. Our knowledge is based on randomised, controlled trials (RCTs) and long-term observations in IPAH with intravenous epoprostenol [16-18, 20] or oral bosentan [29-31]. Long-term data are also available in PAH with ambrisentan [32] and in PAHCTD with bosentan $[29,33-35]$ and sitaxentan $[36,37]$.

\section{Importance of baseline WHO FC}

In one study of patients with IPAH treated with epoprostenol, 3- and 5-yr survival rates in WHO FC IV patients were only $47 \%$ and $27 \%$, compared with $81 \%$ and $70 \%$ for WHO FC III patients [17]. Similar findings in IPAH were also reported by SITBON et al. [18]: 1-, 2- and 3-yr survival rates were 76\%, 60\% and $47 \%$ respectively, in WHO FC IV, compared with $90 \%$, $76 \%$ and $71 \%$, respectively, for WHO FC III patients (fig. 1a). The Kaplan-Meier estimates of survival in these WHO FC IV and WHO FC III patients show separation within $1 \mathrm{yr}$ of treatment. This influence of FC has also been reported in patients with PAH-CTD treated using PAH-specific therapy; risk of death for those in WHO FC III/IV was twice that of those in WHO FC I/II (fig. 1b) [9]. Furthermore, in a study of 49 consecutive patients with PAH-SSc treated with bosentan with or without additional therapies, FC was the only baseline parameter that was found to be significantly related to survival, with an overall survival hazard ratio of 5.60 (95\% CI 4.34-100; $\mathrm{p}=0.0001$ ) for WHO FC IV versus FC II/III patients [35].

\section{Prognostic implications of WHO FC under therapy}

In addition to baseline FC, the level of WHO FC achieved during treatment is also associated with survival. Overall, patients who respond to $\mathrm{PAH}$-specific therapy have a better prognosis than historical controls managed using nonspecific interventions [16, 29, 35]. Patients who remain in, or move into, WHO FC IV with epoprostenol treatment have an especially poor survival rate, while those who have an improved WHO FC have a better prognosis. In the study by SITBON et al. [18], patients who improved to WHO FC I/II after 3 months of treatment with epoprostenol had observed survival rates of $100 \%, 93 \%$ and $88 \%$ at 1,2 and 3 yrs, respectively, compared with $77 \%, 46 \%$ and $33 \%$, respectively, in those patients who remained in WHO FC III/IV (fig. 2a). This study also demonstrated that, although both baseline and on-treatment WHO FC IV was associated with survival on univariate analysis, only persistence of WHO FC III or IV at 3 months of treatment was independently associated with mortality on multivariate analysis. In another long-term study, patients who were in WHO FC IV at the end of the first follow-up period had survival rates of $42 \%$ at 2 yrs and $0 \%$ at 3 yrs, while those in WHO FC I or II had 3- and 5-yr survival rates of $89 \%$ and $73 \%$, respectively (fig. 2b) [17]. These data suggest that ontreatment response in terms of improvement in WHO FC may be a stronger indicator of prognosis than baseline FC. Given the strong influence of WHO FC on prognosis, it is clear that patients in WHO FC IV need aggressive treatment strategies to improve their FC and, therefore, their outcomes.

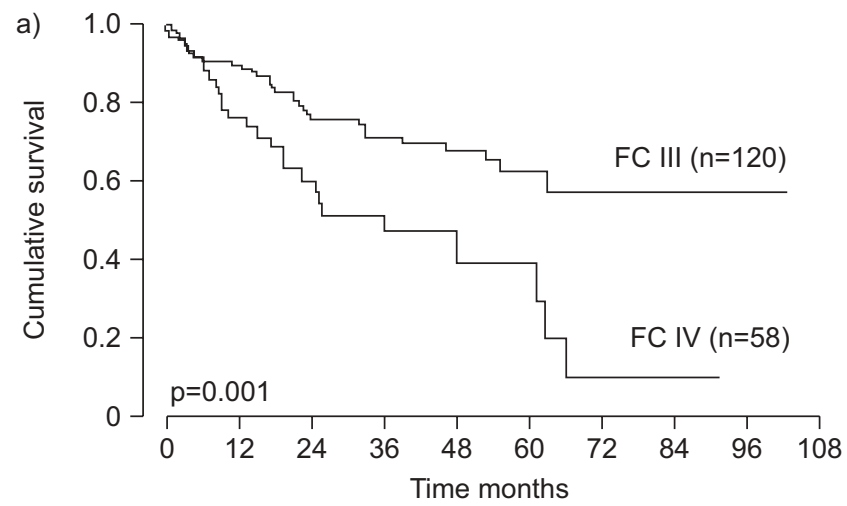

b)

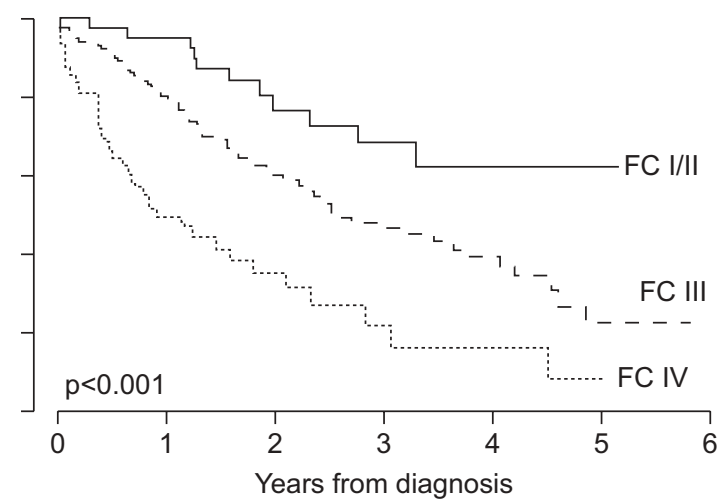

$\begin{array}{ccccccccccc}\text { Subjects } & 120 & 92 & 65 & 46 & 31 & 17 & 6 & 2 & 1 & \text { FC III } \\ \text { at risk n } & 58 & 37 & 20 & 11 & 5 & 4 & 1 & 1 & & \text { FC IV }\end{array}$

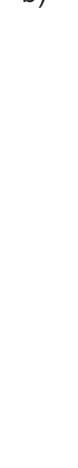

Patients at risk $\mathrm{n} 176$

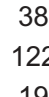

38

19
20

64

10
14
35
4

6

19

2
1 FC I/II

1 FC IV

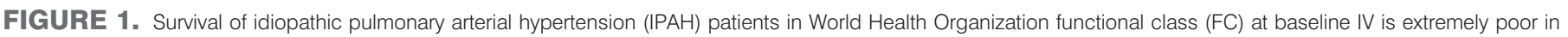

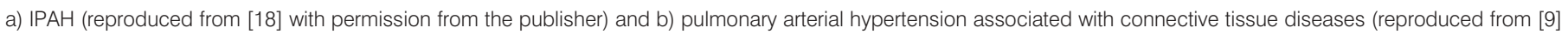
with permission from the publisher). 

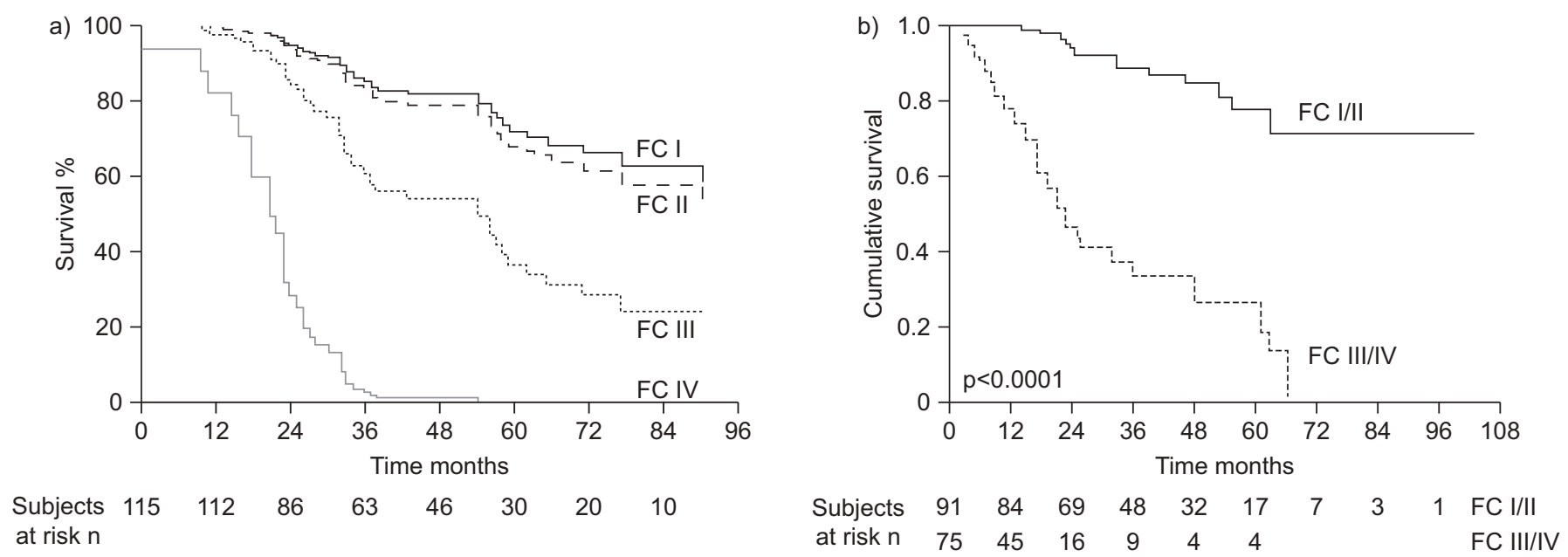

FIGURE 2. Patients who remain in World Health Organization function class (FC) IV after treatment is initiated have a poor prognosis. a) Data from the USA. Reproduced from [17] with permission from the publisher. b) Data from Europe. Reproduced from [18] with permission from the publisher.

\section{EVIDENCE SUPPORTING CURRENT TREATMENT GUIDELINES FOR WHO FC IV}

Although there have been advances in our understanding of the pathophysiology of $\mathrm{PAH}$ in recent years, the exact processes which initiate and drive the underlying changes seen in the pulmonary vasculature have not yet been fully elucidated. However, it is clear that PAH has a multifactorial pathobiology involving a number of major biochemical pathways and cell types [38]. Currently, three of these pathways are targeted by PAH-specific therapies recommended in the current treatment guidelines: endothelin-receptor antagonists, prostacyclin analogues and phosphodiesterase type-5 inhibitors [5].

\section{Patients in WHO FC IV included in clinical trials}

The majority of data in evidence-based guidelines have come from the limited number of trials that have enrolled the highest percentages of WHO FC IV patients. These have predominantly been trials of prostanoid therapy; for example, the pivotal trial of epoprostenol in IPAH, in which $27 \%$ of the 81 patients enrolled were classed as WHO FC IV [20]. The inhaled iloprost AIR trial included 203 patients, $~ 50 \%$ of whom had IPAH, with the remaining 50\% predominantly comprised of patients with PAH-CTD and inoperable CTEPH. Overall, a remarkably high number of patients in the trial (41\%) were classified as being in WHO FC IV [39]. The classification of patients in the AIR study is somewhat controversial as, despite a greater baseline mean 6MWD in the AIR trial compared with the epoprostenol trial discussed above, the proportion of WHO FC IV patients was actually considerably higher in the AIR (Aerosolized Iloprost Randomized) trial [33]. Whether this reflects differences in the classification of patients between trials, or actual baseline differences between the groups, is unclear. In addition, this apparent overrepresentation of patients in WHO FC IV in the AIR trial has never been replicated in any other clinical trial. This may explain, at least in part, why the primary composite endpoint was positive, as patients are more likely to improve from WHO FC IV to III (or even II) than to go from WHO FC III to II (or I).
More recent clinical trials of other types of PAH-specific therapy have included only a small proportion of WHO FC IV patients, often less than $5 \%$ of the total study population (table 2). Given that registry data suggest that up to $24 \%$ of patients are already in WHO FC IV when they are diagnosed $[7,9,24,25]$, it is surprising that clinical trials of treatmentnaïve patients do not include a higher proportion of these severely affected individuals. The low number of patients may reflect the inclusion and exclusion criteria; for example, some trials included only those patients with a stable clinical status [42] or those who fulfilled criteria such as a 6 MWD $>100 \mathrm{~m}$ or $>150 \mathrm{~m}[43,45,47]$. In addition, as evidence has emerged about the poor prognosis of WHO FC IV patients and the improvements in survival seen with agents such as epoprostenol, such patients have been excluded from placebocontrolled trials because of the ethical concern that they could miss out on vital treatment by being enrolled in a placebo group $[41,48]$. Data are, therefore, limited in WHO FC IV patients, and more studies are required to further define and support recommendations in treatment guidelines, especially those regarding strategies other than prostanoid therapy.

\section{Targeted therapy in severe $\mathrm{PAH}$}

Even where trials included WHO FC IV patients, the majority did not specifically detail results in this group. In a pivotal study of WHO FC III and IV patients, treatment with epoprostenol compared with conventional therapy resulted in significant improvements in 6MWD (mean $+32 \mathrm{~m}$ versus $-29 \mathrm{~m}$, respectively; $\mathrm{p}<0.002)$, FC $(40 \%$ versus $3 \%$ improved, respectively; $\mathrm{p}<0.02)$ and haemodynamic parameters, including $\bar{P}_{\text {pa }}(-8 \%$ versus $-3 \%$, respectively; $\mathrm{p}<0.002)$ and PVR $(-21 \%$ versus $-9 \%$, respectively; $\mathrm{p}<0.001)$ [20]. Survival was also significantly improved in epoprostenol-treated patients. WHO FC IV patients made up around one-quarter of the 81 patients included in the study, but as they were not analysed as a separate population, it is not possible to assess their response specifically [20]. In the AIR study, $22.6 \%$ of PAH patients improved by at least one FC following 12 weeks of therapy with inhaled iloprost, although it is unclear whether these 

$\begin{array}{ll}\text { TABLE } 2 & \begin{array}{l}\text { World Health Organization functional class (WHO FC) IV patients in randomised clinical trials of pulmonary arterial } \\ \text { hypertension }\end{array}\end{array}$

\section{Epoprostenol \\ lloprost ${ }^{\#}$ \\ Treprostinil \\ Bosentan \\ Ambrisentan \\ Sitaxentan \\ Sildenafil \\ Tadalafil}

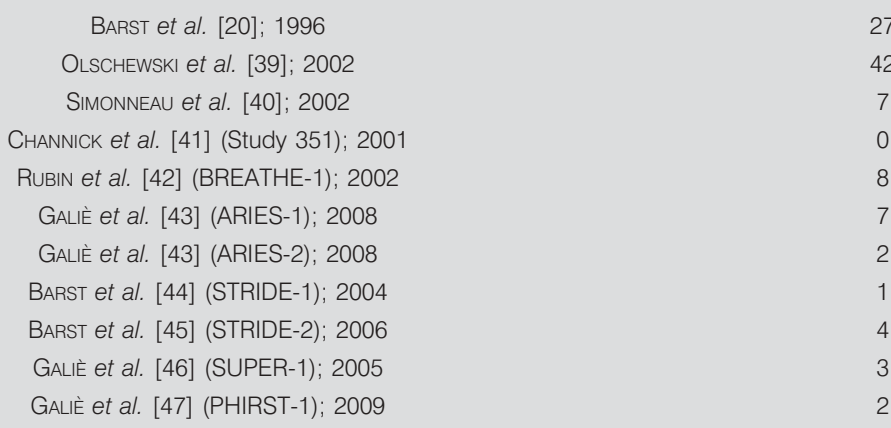

7

1

4

BREATHE: Bosentan Randomized Trial of Endothelin Antagonist Therapy; ARIES: Ambrisentan in Pulmonary Arterial Hypertension, Randomized, Double-Blind, PlaceboControlled, Multicenter, Efficacy Study; STRIDE: Sitaxsentan To Relieve Impaired Exercise; SUPER: Sildenafil Use in Pulmonary Arterial Hypertension; PHIRST: Pulmonary Arterial Hypertension and Response to Tadalafil. \#: patients were stratified by WHO FC prior to being randomised.

improvements were seen predominantly in patients classified as WHO FC III or IV at baseline [39].

One of the few studies of non-prostanoid therapy to include a significant proportion of WHO FC IV patients was the BREATHE (Bosentan Randomized Trial of Endothelin Antagonist Therapy)-2 trial, which examined the combination of epoprostenol with bosentan in WHO FC III/IV patients (24\% WHO FC IV) [38]. The study suggested that the addition of bosentan to epoprostenol was more effective than epoprostenol monotherapy in terms of haemodynamic parameters, although this did not reach statistical significance. Overall, FC improved in four of the five $(80 \%)$ WHO FC IV patients in the combination therapy group, and in one of three $(33 \%)$ in the epoprostenol monotherapy group [38].

\section{SURGICAL INTERVENTION IN END-STAGE PAH}

A certain proportion of PAH patients fail to respond adequately to all forms of medical therapy, including sequential combination therapy. The only management option available to these patients is an invasive procedure, of which two are available: balloon atrial septostomy (BAS) and lung transplantation [5].

\section{Balloon atrial septostomy}

The BAS procedure creates an inter-atrial right-to-left shunt via an operation known as graded balloon dilation atrial septostomy [49, 50]. Utility of BAS was originally inferred from observations that patients with PAH-CHD have better outcomes compared with IPAH patients: in Eisenmenger's syndrome, for example, ventricular function is preserved for longer, and survival is higher among PAH-CHD patients awaiting transplantation [51]. Survival is also increased in IPAH when a patent foramen ovale is present [52].

Prior to BAS, a thorough risk assessment should be performed, and candidates should be receiving optimal medical treatment that may include pre-conditioning with inotropes. The main indication for BAS is severe adult IPAH, although it may also be performed in patients with $\mathrm{PAH}$ and surgically corrected CHD,
PAH-CTD and distal CTEPH [5]. Patients in WHO FC IV, failing on medical therapy and with persistent signs of right ventricular failure and/or recurrent syncope, are also indicated [5, 49, 50]. The procedure should be avoided in end-stage patients with a baseline mean right atrial pressure $>20 \mathrm{mmHg}$ and at-rest oxygen saturation $<80 \%$ on room air [5].

Creation of a shunt by BAS decompresses the right heart chambers, and increases left ventricular preload and cardiac output $[49,50]$. Benefits of the procedure include improved cardiac index, increased systemic oxygen transport despite reduced oxygen saturation, decreased right atrial pressure, reduced neurohumoral activation and improved 6MWD [49, $50,53,54]$. Long-term survival following BAS has not been established in randomised, controlled clinical trials [5, 49, 50], although a recent case series reported median and mean survival of 60 months and 52.3 months, respectively, among $128 \mathrm{PAH}$ patients treated with BAS whose follow-up information was available [55]. Current guidelines describe BAS as a palliative procedure that may act as a bridge to lung transplantation, which should only be performed at centres with specific experience of $\mathrm{PH}[5,55]$.

\section{Lung transplantation}

Lung transplantation remains an important therapeutic option for $\mathrm{PAH}$ patients refractory to medical treatment (that is, patients in WHO FC IV or those in WHO FC III despite combination treatment) despite a reduction in demand since the advent of PAH-specific drugs [5]. The differential outcomes of the various forms of PAH affect the timing of transplantation. Patients with WHO FC IV IPAH who may be eligible for transplantation should be immediately referred for assessment and, because of their worse prognosis, transplantation should be considered earlier for PAH-CTD patients [55]. Eisenmenger's syndrome and congenital left-to-right shunts have a better prognosis, but the decision to transplant should not be delayed until the advent of renal and hepatic failure [55]. In general, current guidelines state that patients should be placed on the lung transplantation waiting list if they display 
clinical parameters predictive of a worse prognosis, despite maximal medical therapy [5]. Features include, but are not limited to, syncope, $6 \mathrm{MWD}<300 \mathrm{~m}$, rapid symptom progression, presence of right ventricular failure, and poor haemodynamic and echocardiographic results [5].

Transplantation for PAH may take the form of either heartlung (HLT) or double-lung transplantation (DLT), with DLT the predominant procedure, due to factors such as a lack of donor organs and local allocation protocols [5, 55]. The HLT procedure offers survival advantages in patients with congenital cardiac abnormalities and Eisenmenger's syndrome, however, in whom it is the operation of choice [55, 56]. Individual centres have developed their own protocols for selecting which type of transplantation patients should receive, largely because the unrecoverable threshold of right ventricular systolic dysfunction has not been defined [5].

The immediate effect of DLT is to reduce right ventricular afterload, but since neither right ventricular systolic nor diastolic functions immediately reduce, haemodynamic instability is common soon after the procedure [5]. Evidence shows that QoL and exercise capacity are significantly improved by lung transplantation [55,57], but this is achieved at the cost of the lowest 3-month survival of all lung transplant recipients [57]. However, good long-term survival is achieved with both HLT and DLT: a recent study of $219 \mathrm{PH}$ patients, of whom 147 had PAH, reported 5-, 10- and 15-yr survival rates of 50\%, 39\% and $26 \%$, respectively, for HLT and $52 \%, 43 \%$ and $30 \%$, respectively, for DLT [58].

\section{TREATMENT STRATEGIES: PRESENT AND FUTURE}

Based on the available data, as described above, the recently revised European Society of Cardiology and European Respiratory Society treatment guidelines give first-line intravenous epoprostenol monotherapy the highest recommendation (IA) for WHO FC IV patients, because of the demonstrated survival benefit in these patients [5]. Other therapies, including iloprost (inhaled and intravenous), treprostinil (subcutaneous, intravenous and inhaled), ambrisentan, bosentan, sitaxentan, sildenafil and tadalafil were given a grade IIa-C recommendation in the guidelines (fig. 3) [5]. This recommendation reflects both the lack of available data for these agents and the fact that data from single-centre, retrospective studies are not as highly valued as findings from RCTs.

At present, the use of a goal-oriented approach to therapy is recommended [5], the aim of which is to improve and/or maintain values of proven prognostic indicators, such as WHO FC, 6MWD and haemodynamics, to levels associated with improved outcomes. If the treatment goals are not met and response is inadequate on first-line therapy, it is recommended that treatment be escalated and sequential combination therapy considered. For patients in WHO FC IV, inadequate response is defined as a lack of rapid improvement to WHO FC III or better, or a "stable and not satisfactory" status where, although their disease is stable, the patient "has not achieved the status which patient and treating physician would consider desirable". As part of this goal-oriented management approach, patients require careful and regular assessment, particularly after initiation of, or change in, therapy. This is achieved using a number of clinical, invasive and noninvasive techniques to accurately monitor and assess response and to define the patient's clinical condition. Such assessments should ideally be carried out in specialist centres, as should treatment, especially if combination therapy is required. For the first time, combination therapy (double or triple) is suggested as a possible firstline strategy in the new guidelines, with the same class of recommendation and level of evidence as monotherapy with agents other than epoprostenol [5]. However, there are a great many factors that need to be resolved regarding combination therapy, whether it is to be used as sequential or first-line treatment.

Given the multifactorial nature of $\mathrm{PAH}$, the co-administration of agents that target different pathways is scientifically rational and, despite the lack of supporting evidence, anecdotal reports and personal experience suggest that combination therapy is widely used in many PAH centres, particularly for the treatment of more severely ill patients. Data from numerous case studies and several small clinical trials have shown that various drug combinations appear to be safe and effective, although, again, there are limited data specific to WHO FC IV. In 123 stable WHO FC III/IV patients $(80 \% / 20 \%)$, the use of a goal-oriented approach to combination therapy, where sildenafil and then inhaled iloprost were added sequentially to firstline bosentan therapy if treatment goals were not achieved, improved survival relative to expected and historical controls, and reduced the need for epoprostenol and lung transplantation [59]. Combination therapy was well tolerated, with no patient showing signs of additive toxicity. The BREATHE-2 trial of epoprostenol and bosentan combination therapy in WHO FC III and IV patients (24\% WHO FC IV) demonstrated a non-statistically significant trend toward greater haemodynamic and clinical improvement in the combination arm, compared with epoprostenol monotherapy [38]. In this study, patients were treated initially with epoprostenol for 2 days prior to randomisation to either bosentan or placebo, effectively representing first-line combination therapy. More

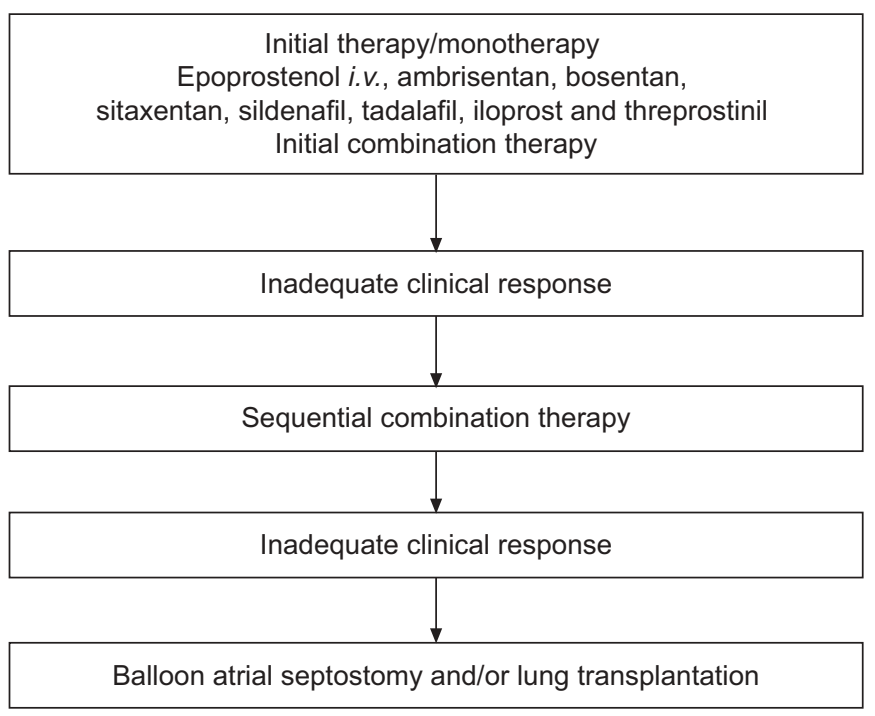

FIGURE 3. Evidence-based treatment of World Health Organization functional class IV pulmonary arterial hypertension patients. Modified from [5] with permission from the publisher. 
recently, bosentan and epoprostenol given as first-line therapy to 23 patients in WHO FC III and IV (70\% and 30\%, respectively) has also shown encouraging results [60]. After 1 month of combination therapy, none of the patients remained in WHO FC IV, and almost 50\% had achieved WHO FC II, with concomitant significant improvements in 6MWD and haemodynamic parameters.

Although clearly a promising strategy, there are a number of outstanding issues regarding combination therapy, including which combination of agents to use, when to combine agents (i.e. as first-line treatment or a sequential combination based on response), the optimal duration of combination therapy and the relevant dosing of agents in combination to maximise efficacy while minimising side-effects. It is possible that "firstline" combination therapy may involve not treating with all agents at once, but rather adding each sequentially over a short period of time to manage side-effects, as occurs in other clinical conditions. For example, in heart failure, patients are first stabilised on angiotensin-converting enzyme inhibitors before beta-blockers are given; further agents, such as aldosterone antagonists, are then added if the patient's signs and symptoms persist [61]; such escalation of therapy may also turn out to be the optimal strategy in PAH. Whatever strategy is used, the management of patients with $\mathrm{PAH}$, and particularly those in WHO FC IV who may show rapid disease progression, must be done in expert centres, with a close association between clinicians and cardiac surgical teams should problems arise. This is particularly true of BAS and transplantation for end-stage $\mathrm{PAH}$ patients, which are complex operations that require the experience of dedicated $\mathrm{PH}$ centres to minimise complications. Their key role in the overall management of PAH is recognised by a level IC recommendation in the current guidelines [5].

\section{CONCLUSIONS}

FC is a simple tool to assess patients' disease severity and a powerful marker of prognosis. The outcome of the significant proportion of patients who present with severe, WHO FC IV disease is dismal, with rapid progress to end-stage disease and death in the absence of treatment. In addition, rapid improvement in FC is associated with a better prognosis. Therefore, severe PAH clearly requires rapid and aggressive treatment in order to achieve this in the shortest possible time frame. Current treatment guidelines recommend rapid, sequential combination therapy based on the achievement of predefined clinical goals, and first-line combination therapy may also be considered. Data in support of new strategies, such as combination therapy, in this patient group are lacking, and there is an obvious need for studies that address the specific issues associated with such a severely ill population. These data should be provided by strategy trials aimed at improving management and outcome, with decreased emphasis on trials that are primarily designed to gain regulatory approval for individual agents, particularly when those agents are similar to those already available. The design and implementation of such strategy trials will raise considerable challenges, not least in the selection of suitable and ethical regimens for the treatment and control arms. In addition, the number of patients required to achieve statistical significance would be high; in PAH, this would require multinational studies, raising issues around discrepancies in the currently favoured methodologies and the availability of certain agents. Nevertheless, combination therapy is already widely used in clinical practice, and such trials are clearly required to support, improve and refine this approach, particularly for severely ill WHO FC IV patients. These patients continue to make up a significant proportion of newly diagnosed cases and continue to have a poor prognosis, despite the major advances seen in the treatment of $\mathrm{PAH}$ over recent years. If we are to improve outcome for this group, it may be time for a shift in paradigm to early and aggressive treatment using new, possibly more complex, treatment strategies.

\section{STATEMENT OF INTEREST}

J-L. Vachiéry has received speaker's fee and travel grants from Actelion Pharmaceuticals, GlaxoSmithKline, Pfizer, United Therapeutics and Bayer Schering; consultancy fees from GlaxoSmithKline, Pfizer, United Therapeutics and Elli Lilly; and research grants from United Therapeutics.

\section{ACKNOWLEDGEMENTS}

We received editorial assistance from L. Thomas, Elements Communications Ltd (Westerham, UK), supported by Actelion Pharmaceuticals Ltd (Allschwil, Switzerland).

\section{REFERENCES}

1 Galiè N, Rubin LJ, Hoeper MM, et al. Treatment of patients with mildly symptomatic pulmonary arterial hypertension with bosentan (EARLY study): a double-blind, randomised controlled trial. Lancet 2008; 371: 2093-2100.

2 McLaughlin VV, Presberg KW, Doyle RL, et al. Prognosis of pulmonary arterial hypertension: ACCP evidence-based clinical practice guidelines. Chest 2004; 126: Suppl. 1, 78S-92S.

3 Kawut SM, Taichman DB, Archer-Chicko CL, et al. Hemodynamics and survival in patients with pulmonary arterial hypertension related to systemic sclerosis. Chest 2003; 123: 344-350.

4 Barst RJ, Gibbs JS, Ghofrani HA, et al. Updated evidence-based treatment algorithm in pulmonary arterial hypertension. J Am Coll Cardiol 2009; 54: Suppl. 1, S78-S84.

5 Galiè N, Hoeper MM, Humbert M, et al. Guidelines for the diagnosis and treatment of pulmonary hypertension: The Task Force for the Diagnosis and Treatment of Pulmonary Hypertension of the European Society of Cardiology (ESC) and the European Respiratory Society (ERS), endorsed by the International Society of Heart and Lung Transplantation (ISHLT). Eur Heart J 2009; 30: 2493-2537.

6 Simonneau G, Robbins IM, Beghetti M, et al. Updated clinical classification of pulmonary hypertension. J Am Coll Cardiol 2009; 54: Suppl. 1, S43-S54.

7 Humbert M, Sitbon O, Chaouat A, et al. Pulmonary arterial hypertension in France: results from a national registry. Am J Respir Crit Care Med 2006; 173: 1023-1030.

8 Peacock AJ, Murphy NF, McMurray JJ, et al. An epidemiological study of pulmonary arterial hypertension. Eur Respir J 2007; 30: 104-109.

9 Condliffe R, Kiely DG, Peacock AJ, et al. Connective tissue diseaseassociated pulmonary arterial hypertension in the modern treatment era. Am J Respir Crit Care Med 2009; 179: 151-157.

10 Hachulla E, Gressin V, Guillevin L, et al. Early detection of pulmonary arterial hypertension in systemic sclerosis: a French nationwide prospective multicenter study. Arthritis Rheum 2005; 52: 3792-3800.

11 Mukerjee D, St George D, Coleiro B, et al. Prevalence and outcome in systemic sclerosis associated pulmonary arterial hypertension: 
application of a registry approach. Ann Rheum Dis 2003; 62: 1088-1093.

12 Haas C. Pulmonary hypertension associated with systemic lupus erythematosus. Bull Acad Natl Med 2004; 188: 985-997.

13 Rich S. Executive summary from the World Symposium on Primary Pulmonary Hypertension 1998. World Health Organization. http:// web.archive.org/web/20020408173726/http:/ /www.who.int/ncd/ cvd/pph.html. Date last updated: November 21, 2001. Date last accessed: May 17, 2010.

14 Barst RJ, McGoon M, Torbicki A, et al. Diagnosis and differential assessment of pulmonary arterial hypertension. J Am Coll Cardiol 2004; 43, 12: Suppl. S, 40S-47S.

15 D'Alonzo GE, Barst RJ, Ayres SM, et al. Survival in patients with primary pulmonary hypertension. Results from a national prospective registry. Ann Intern Med 1991; 115: 343-349.

16 Barst RJ, Rubin LJ, McGoon MD, et al. Survival in primary pulmonary hypertension with long-term continuous intravenous prostacyclin. Ann Intern Med 1994; 121: 409-415.

17 McLaughlin VV, Shillington A, Rich S. Survival in primary pulmonary hypertension: the impact of epoprostenol therapy. Circulation 2002; 106: 1477-1482.

18 Sitbon $\mathrm{O}$, Humbert $\mathrm{M}$, Nunes $\mathrm{H}$, et al. Long-term intravenous epoprostenol infusion in primary pulmonary hypertension: prognostic factors and survival. J Am Coll Cardiol 2002; 40: 780-788.

19 Cenedese E, Speich R, Dorschner L, et al. Measurement of quality of life in pulmonary hypertension and its significance. Eur Respir J 2006; 28: 808-815.

20 Barst RJ, Rubin LJ, Long WA, et al. A comparison of continuous intravenous epoprostenol (prostacyclin) with conventional therapy for primary pulmonary hypertension. The Primary Pulmonary Hypertension Study Group. N Engl J Med 1996; 334: 296-302.

21 McKenna SP, Doughty N, Meads DM, et al. The Cambridge Pulmonary Hypertension Outcome Review (CAMPHOR): a measure of health-related quality of life and quality of life for patients with pulmonary hypertension. Qual Life Res 2006; 15: 103-115.

22 Shafazand S, Goldstein MK, Doyle RL, et al. Health-related quality of life in patients with pulmonary arterial hypertension. Chest 2004; 126: 1452-1459.

23 Taichman DB, Shin J, Hud L, et al. Health-related quality of life in patients with pulmonary arterial hypertension. Respir Res 2005; 6: 92.

24 Rubenfire M, Lippo G, Bodini BD, et al. Evaluating health-related quality of life, work ability, and disability in pulmonary arterial hypertension: an unmet need. Chest 2009; 136: 597-603.

25 Jing ZC, Xu XQ, Han ZY, et al. Registry and survival study in Chinese patients with idiopathic and familial pulmonary arterial hypertension. Chest 2007; 132: 373-379.

26 Tueller C, Stricker H, Soccal P, et al. Epidemiology of pulmonary hypertension: new data from the Swiss registry. Swiss Med Wkly 2008; 138: 379-384.

27 Sztrymf B, Souza R, Bertoletti L, et al. Prognostic factors of acute heart failure in patients with pulmonary arterial hypertension. Eur Respir J 2010; 35: 1286-1293.

28 Kearon C, Kahn SR, Agnelli G, et al. Antithrombotic therapy for thromboembolic disease: American College of Chest Physicians Evidence-Based Clinical Practice Guidelines (8th Edition). Chest 2008; 133: Suppl. 6, 454S-545S.

29 McLaughlin VV. Survival in patients with pulmonary arterial hypertension treated with first-line bosentan. Eur J Clin Invest 2006; 36: Suppl. 3, 10-15.

30 McLaughlin VV, Sitbon O, Badesch DB, et al. Survival with firstline bosentan in patients with primary pulmonary hypertension. Eur Respir J 2005; 25: 244-249.

31 Sitbon O, McLaughlin VV, Badesch DB, et al. Survival in patients with class III idiopathic pulmonary arterial hypertension treated with first line oral bosentan compared with an historical cohort of patients started on intravenous epoprostenol. Thorax 2005; 60: 1025-1030.

32 Oudiz R, Galiè N, Olschewski H, et al. Long-term ambrisentan therapy for the treatment of pulmonary arterial hypertension. J Am Coll Cardiol 2009; 54: 1971-1981.

33 Denton $\mathrm{CP}$, Humbert M, Rubin L, et al. Bosentan therapy for pulmonary arterial hypertension related to connective tissue disease: a subgroup analysis of the pivotal clinical trials and their open-label extensions. Ann Rheum Dis 2006; 65: 1336-1340.

34 Denton CP, Pope JE, Peter HH, et al. Long-term effects of bosentan on quality of life, survival, safety and tolerability in pulmonary arterial hypertension related to connective tissue diseases. Ann Rheum Dis 2008; 67: 1222-1228.

35 Launay D, Sitbon O, Le Pavec J, et al. Long-term outcome of systemic sclerosis-associated pulmonary arterial hypertension treated with bosentan as first-line monotherapy followed or not by the addition of prostanoids or sildenafil. Rheumatology 2010; 49: 490-500.

36 Benza RL, Barst RJ, Galie N, et al. Sitaxsentan for the treatment of pulmonary arterial hypertension: a 1-year, prospective, open-label observation of outcome and survival. Chest 2008; 134: 775-782.

37 Seibold JR, Benza R, Davie N, et al. Long term results of sitaxentan therapy in pulmonary arterial hypertension $(\mathrm{PAH})$ associated with connective tissue disease. Ann Rheum Dis 2009; 68: Suppl. 3, 462.

38 Humbert M, Barst RJ, Robbins IM, et al. Combination of bosentan with epoprostenol in pulmonary arterial hypertension: BREATHE-2. Eur Respir J 2004; 24: 353-359.

39 Olschewski H, Simonneau G, Galiè N, et al. Inhaled iloprost for severe pulmonary hypertension. N Engl J Med 2002; 347: 322-329.

40 Simonneau G, Barst RJ, Galiè N, et al. Continuous subcutaneous infusion of treprostinil, a prostacyclin analogue, in patients with pulmonary arterial hypertension: a double-blind, randomized, placebo-controlled trial. Am J Respir Crit Care Med 2002; 165: 800-804.

41 Channick RN, Simonneau G, Sitbon O, et al. Effects of the dual endothelin-receptor antagonist bosentan in patients with pulmonary hypertension: a randomised placebo-controlled study. Lancet 2001; 358: 1119-1123.

42 Rubin LJ, Badesch DB, Barst RJ, et al. Bosentan therapy for pulmonary arterial hypertension. N Engl J Med 2002; 346: 896-903.

43 Galiè N, Olschewski H, Oudiz RJ, et al. Ambrisentan for the treatment of pulmonary arterial hypertension: results of the ambrisentan in pulmonary arterial hypertension, randomized, double-blind, placebo-controlled, multicenter, efficacy (ARIES) study 1 and 2. Circulation 2008; 117: 3010-3019.

44 Barst RJ, Langleben D, Frost A, et al. Sitaxsentan therapy for pulmonary arterial hypertension. Am J Respir Crit Care Med 2004; 169: 441-447.

45 Barst RJ, Langleben D, Badesch D, et al. Treatment of pulmonary arterial hypertension with the selective endothelin-A receptor antagonist sitaxsentan. J Am Coll Cardiol 2006; 47: 2049-2056.

46 Galiè N, Ghofrani HA, Torbicki A, et al. Sildenafil citrate therapy for pulmonary arterial hypertension. $N$ Engl J Med 2005; 353: 2148-2157.

47 Galiè N, Brundage BH, Ghofrani HA, et al. Tadalafil therapy for pulmonary arterial hypertension. Circulation 2009; 119: 2894-2903.

48 Hill NS, Preston IR, Roberts KE. Patients with pulmonary arterial hypertension in clinical trials: who are they? Proc Am Thorac Soc 2008; 5: 603-609.

49 Kurzyna M, Dabrowski M, Bielecki D, et al. Atrial septostomy in treatment of end-stage right heart failure in patients with pulmonary hypertension. Chest 2007; 131: 977-983.

50 Sandoval J, Gaspar J, Pulido T, et al. Graded balloon dilation atrial septostomy in severe primary pulmonary hypertension. A therapeutic alternative for patients nonresponsive to vasodilator treatment. J Am Coll Cardiol 1998; 32: 297-304.

51 Hopkins WE, Ochoa LL, Richardson GW, et al. Comparison of the hemodynamics and survival of adults with severe primary 
pulmonary hypertension or Eisenmenger syndrome. J Heart Lung Transplant 1996; 15: 100-105.

52 Rozkovec A, Montanes P, Oakley CM. Factors that influence the outcome of primary pulmonary hypertension. Br Heart J 1986; 55: 449-458.

53 Reichenberger F, Pepke-Zaba J, McNeil K, et al. Atrial septostomy in the treatment of severe pulmonary arterial hypertension. Thorax 2003; 58: 797-800.

54 Ciarka A, Vachièry J-L, Houssière A, et al. Atrial septostomy decreases sympathetic overactivity in pulmonary arterial hypertension. Chest 2007; 131: 1831-1837.

55 Keogh A, Benza RL, Corris $\mathrm{P}$, et al. Interventional and surgical modalities of treatment in pulmonary arterial hypertension. J Am Coll Cardiol 2009; 54: S67-S77.

56 Waddell TK, Bennett L, Kennedy R, et al. Heart-lung or lung transplantation for Eisenmenger syndrome. J Heart Lung Transplant 2002; 21: 731-737.

57 Christie JD, Edwards LB, Aurora P, et al. Registry of the International Society for Heart and Lung Transplantation: twenty-fifth official adult lung and heart/lung transplantation report-2008. J Heart Lung Transplant 2008; 27: 957-969.

58 Fadel E, Mercier O, Mussot S, et al. Long-term outcome of doublelung and heart-lung transplantation for pulmonary hypertension: a comparative retrospective study of 219 patients. Eur J Cardiothoracic Surg 2010; 38: 277-284.

59 Hoeper MM, Markevych I, Spiekerkoetter E, et al. Goal-oriented treatment and combination therapy for pulmonary arterial hypertension. Eur Respir J 2005; 26: 858-863.

60 Kemp K, Sitbon O, Jais X, et al. Initial combination therapy with epoprostenol and bosentan in severe pulmonary arterial hypertension. Eur Respir J 2009; 34: Suppl. 53, E1496.

61 ESC Guidelines for the diagnosis and treatment of acute and chronic heart failure 2008: the Task Force for the Diagnosis and Treatment of Acute and Chronic Heart Failure 2008 of the European Society of Cardiology. Developed in collaboration with the Heart Failure Association of the ESC (HFA) and endorsed by the European Society of Intensive Care Medicine (ESICM). Eur Heart J 2008; 29: 2388-2442. 